\title{
EXTENSION OF NONLINEAR CONTRACTIONS
}

\author{
BY STEN OLOF SCHÖNBECK
}

Communicated by W. Rudin, September 24, 1965

The following problem was suggested as a research problem by R. A. Hirschfeld in Bull. Amer. Math. Soc. 71 (1965), 495:

$E$ and $F$ are Banach spaces, $F$ reflexive, $D$ is a subset of $E$ and $T: D \rightarrow F$ a nonlinear contraction, i.e. $\left\|T x_{1}-T x_{2}\right\| \leqq\left\|x_{1}-x_{2}\right\|$ whenever $x_{1}, x_{2} \in D$. Can $T$ be extended to a contraction $T^{\prime}: E \rightarrow F$ ?

Hirschfeld observes that the answer is "yes" when $E=F=$ Hilbert space.

The following simple example shows that the answer is "no" in general. In the two-dimensional plane $R^{2}$ consider a regular hexagon $H$, with its center at the origin, and a circle $C$ inscribed in $H$. Let $E$ and $F$ be $R^{2}$ equipped with norms $\|\cdot\|_{E}$ and $\|\cdot\|_{F}$ defined by $\left\{x ;\|x\|_{E}\right.$ $=1\}=H,\left\{x ;\|x\|_{F}=1\right\}=C$. Let $x_{1}$ and $x_{2}$ be two consecutive points of contact between $H$ and $C$. Then

$$
\left\|x_{1}\right\|_{E}=\left\|x_{1}\right\|_{F}=\left\|x_{2}\right\|_{E}=\left\|x_{2}\right\|_{F}=\left\|x_{1}-x_{2}\right\|_{E}=\left\|x_{1}-x_{2}\right\|_{F}=1
$$

so that if $D=\left\{0, x_{1}, x_{2}\right\}$ and $T(0)=0, T x_{1}=x_{1}, T x_{2}=x_{2}, T$ is a contraction of $D$ into $F$. Now, if $z=\left(x_{1}+x_{2}\right) / 3$, it is easily seen that $\|z\|_{E}=\left\|z-x_{1}\right\|_{E}=\left\|z-x_{2}\right\|_{E}=1 / 2$. Hence, if $T$ could be extended to a contraction $T^{\prime}: E \rightarrow F$, then the point $u=T^{\prime} z$ would satisfy

$$
\|u\|_{F} \leqq 1 / 2, \quad\left\|u-x_{1}\right\|_{F} \leqq 1 / 2, \quad\left\|u-x_{2}\right\|_{F} \leqq 1 / 2
$$

which is clearly impossible.

We have, however, been able to prove some positive results. In order to state these results, we introduce the following terminology. If $E$ and $F$ are normed linear spaces, we say that $(E, F)$ has the contraction-extension (c.e.) property if : for any subset $D \subset E$ and any contraction $T: D \rightarrow F$ there is an extension of $T$ to a contraction $T^{\prime}: E \rightarrow F$.

We then have

THEOREM 1. If $E$ and $F$ are real or complex Banach spaces, if $F$ is strictly convex and if $(E, F)$ has the c.e. property, then $E$ and $F$ are Hilbert spaces.

OUTLINE OF PROOF. It is clearly sufficient to assume that $E$ and $F$ are real spaces. Using the strict convexity of $F$, it is then easy to show that, if $x, y \in E, u, v \in F$ and if $\|x\|=\|u\|,\|y\|=\|v\|,\|x-y\|$ $=\|u-v\|$, then $\|a x+b y\| \geqq\|a u+b v\|$ for all real numbers $a, b$. 
If $x$ and $y$ are elements or a real normed linear space, we say that $x$ is normal to $y$ if $\|x+a y\| \geqq\|x\|$ for all real numbers $a$, and then we write $x N y$. Using our above result and a limiting process we may prove: if $x, y \in E, u, v \in F$ and if $\|x\|=\|u\|,\|y\|=\|v\|, x N y, u N v$, then $\|a x+b y\| \geqq\|a u+b v\|$ for all $a, b$.

With the aid of this result, it is now possible to show that normality is a symmetric relation in both $E$ and $F$. Day [2] has given a construction of all two-dimensional spaces with symmetry of normality. By means of this construction and our previous results we may conclude: if $x, y \in E, u, v \in F$ and if $\|x\|=\|u\|,\|y\|=\|v\|, x N y, u N v$, then $\|a x+b y\|=\|a u+b v\|$ for all $a, b$. This implies that both $E$ and $F$ have the following property, formulated for a normed linear space $L$ :

There is a single-valued function $f$ of two real variables so that for any $x, y \in L$ such that $x N y$ we have $\|x+y\|=f(\|x\|,\|y\|)$.

But this property is characteristic of euclidean (i.e. prehilbert) spaces, as can be shown in a number of ways. (See for instance Hopf [4], where this is shown even without assuming symmetry of the norm.)

Theorem 2. The following two properties of a real Banach space $F$ are equivalent:

(i) $(E, F)$ has the c.e. property for every real Banach space $E$

(ii) any family of closed spheres in $F$, such that any two members of it intersect, has a nonempty intersection.

OUTLINE OF PROOF. (i) $\Rightarrow$ (ii) is proved by first observing that, for any set $S$, the Banach space $m(S)$ of all bounded real-valued functions on $S$ with the supremum norm, has property (ii). We then embed $F$ isometrically in a suitable $m(S)$. If $\left(S_{i}\right), i \in I$, are closed spheres in $F$ such that $S_{i} \cap S_{j} \neq \varnothing$ for all $i, j$, then for the corresponding spheres $\sum_{i}$ in $m(S)$ we have $\bigcap_{i} \sum_{i} \neq \varnothing$. Using the c.e. property of $(m(S), F)$ we then conclude that $\bigcap_{i} S_{i} \neq \varnothing$.

(ii) $\Rightarrow$ (i) is proved by Zorn's lemma in a straightforward way.

Theorem 2 shows the intimate connection between our present problem and the problem of linear, norm-preserving extension of continuous linear transformations. In fact, it has been proved by Nachbin [6] that property (ii) for a real Banach space $F$ is equivalent to

(iii) for any real Banach space $E$, any closed linear subspace $S$ of $E$ and any continuous linear transformation $T$ of $S$ into $F$, there exists a linear extension $T^{\prime}$ of $T$ to $E$ with values in $F$ and $\left\|T^{\prime}\right\|$ $=\|T\|$. 
Moreover, through the work of Aronszajn-Panitchpakdi [1], Goodner [3], Kelley [5] and Nachbin, it is also known that a real space $F$ has property (iii) if and only if $F$ is linearly isometric to a space $C(S)$, the space of real-valued continuous functions on a compact, Hausdorff and extremally disconnected space $S$. (For a survey of these and related problems, see Nachbin [7].)

Thus we have the following

Corollary to Theorem 2. If $F$ is a real Banach space, then $(E, F)$ has the c.e. property for every real Banach space $E$ if and only if $F$ is linearly isometric to a space $C(S)$, where $S$ is compact, Hausdorff and extremally disconnected.

Finally, using the corollary it is easy to show that a complex Banach space $F$ can never have property (ii). Hence we may conclude that there is no complex Banach space $F$ such that $(E, F)$ has the c.e. property for every complex Banach space $E$.

\section{REFERENCES}

1. N. Aronszajn and P. Panitchpakdi, Extension of uniformly continuous transformations and hyperconvex metric spaces, Pacific J. Math. 6 (1956), 405-439.

2. M. M. Day, Some characterizations of inner-product spaces, Trans. Amer. Math. Soc. 62 (1947), 320-337.

3. D. B. Goodner, Projections in normed linear spaces, Trans. Amer. Math. Soc. 69 (1950), 89-108.

4. E. Hopf, Zur Kennzeichnung der Euklidischen Norm, Math. Z. 72 (1959), 76-81.

5. J. L. Kelley, Banach spaces with the extension property, Trans. Amer. Math. Soc. 72 (1952), 323-326.

6. L. Nachbin, A theorem of the Hahn-Banach type for linear transformations, Trans. Amer. Math. Soc. 68 (1950), 28-46.

7. - Some problems in extending and lifting continuous linear transformations, Proc. International Symposium on Linear Spaces, Jerusalem, 1960, pp. 340-350.

UNIVERSITY OF STOCKHOLM, SWEdEN 